\title{
DISTRIBUTED TEAM FORMATION FOR HUMANOID ROBOT SOCCER
}

\author{
Onuralp Ulusoy and Sanem Sariel-Talay \\ Department of Computer Engineering, Istanbul Technical University, Istanbul, Turkey \\ \{oulusoy, sariel\}@itu.edu.tr
}

\begin{abstract}
Keywords: RoboCup Competitions : Humanoid Robot Soccer : Team Strategy : Multi-Robot Cooperation : Distributed Team Formation.

Abstract: In this paper, we propose an adaptive team formation strategy for humanoid robot soccer. The proposed strategy involves distributed cooperative decisions through both communication and observations. Two agent groups, namely defenders and attackers, are formed by a case-based group formation method. Attackers are formed for constructing an attacking formation around the ball and scoring a goal whenever possible while defenders are for blocking and constructing a defensive obstacle against the opponent team. Cooperative decisions are made using communication among team members. Distribution of agents on the field is ensured by Voronoi cell construction of each agent through observations in a distributed manner. Experiments are set in the RoboCup 3D Soccer Simulation League environment where our method is compared to earlier team formation methods. The results illustrate that a distributed Voronoi cell construction method combined with a case-based grouping algorithm outperforms the others. Furthermore, it has been shown that our method is also robust to communication failures.
\end{abstract}

\section{INTRODUCTION}

RoboCup competitions provide convenient tools to test and validate multi-agent team strategies. Specifically, simulation competitions are suitable for analyzing complicated team strategies in the face of realistic constraints such as limitations on observability, communication and teleoperation. This paper presents a team formation approach for humanoid soccer teams which deal with both competition and cooperation issues. Just like real soccer games, the main objective of a humanoid soccer team is scoring goals against an opponent team. Efficiency of cooperation is an important key factor to win a game. There are mainly two behaviors which involve cooperation issues, namely, passing the ball to a teammate or spreading out to the field of play to gain control of the ball whenever needed. Both behaviors require agents to be in appropriate positions to achieve the desired outcomes. These positions usually belong to special formations which may dynamically change their shapes for different situations during a game. The performance of the overall team is highly dependent on these adaptive formations and the corresponding positions of robotic agents. Contrary to human soccer games, there are not generic formations for humanoid soccer especially because these are also dependent on the underlying motion model. Therefore, the set of mobility constraints of a team plays an important role in the selection of an appropriate team strategy.

We propose an adaptive team formation strategy which can be applied to robot soccer. However, the focus of this paper is on the RoboCup 3D simulated humanoid soccer competitions. Our team strategy is used in the top layer of the software for team beeStanbul (Asta et al., 2011) for RoboCup 3D Soccer Simulation League (SSL). Experiments are set in the RoboCup 3D SSL Environment, Simspark (Simspark Official Website, 2011). Simspark provides an environment for multiplayer soccer games of two competing teams of simulated autonomous humanoid agents (RoboCup 2011 3D Simulation League hosted $9 \times 9$ agent games on a $21 \times 14 \mathrm{~m}$ field.). The team scores more goals in a ten-minute-long match wins the game. Simspark uses ODE (Open Dynamics Engine) for physical agent simulation of Nao humanoid robots by 
Aldebaran Robotics (Aldebaran Robotics Official Website, 2011). The real Nao robot has a height of $57 \mathrm{~cm}$, a weight of $4,5 \mathrm{~kg}$ and 22 degrees of freedom. The robot is equipped with special sensors including a gyroscope, an accelerometer and a force resistance perceptor on each foot. Simspark can simulate all these features and model some realistic limitations including sensor and actuator noise. The simulator also provides limited communication among robots through special effectors and perceptors and visual information in the form of noisy distance and angle values for the objects in the viewpoint of agents.

Some rules of humanoid soccer are different from real soccer due to the limitations of mobility of agents. At present, fouls are not penalized in the RoboCup 3D SSL but crowding the ball. According to the crowding rule, at most two players are allowed to be in the $0.8 \mathrm{~m}$ radius circle around the ball; only a single player from a team in a circle with a radius of $0.4 \mathrm{~m}$ and at most two teammates in a circle with a radius of $1 \mathrm{~m}$. Failure to comply with either of these rules results in a repositioning of an agent out of the field. All these rules should be taken into account in the team strategy for avoiding any penalties.

Our proposed approach considers the mentioned rules to escape from penalties. According to our strategy, robots can be in four different roles, namely, goalkeeper, defender, midfielder and forward. Forward and goalkeeper roles have their own planners. Midfielder and defender roles share the same planner but they differ in positioning on the field. Goalkeeper is a static role which is assigned to an agent for the entire match. The forward role is assigned dynamically based on a voting mechanism through communication. Each agent sends its time cost to be able to control the ball and determine whether it can be in the forward role based on the information from the incoming messages and a selfcalculation.

Two groups (attackers and defenders) are formed with a case-based group formation method and the remaining roles are assigned based on the messages from the team's captain (goalkeeper is selected as the captain due to its widest viewpoint). When there is a failure in communication, agents decide on their roles based on only observations.

The attackers group involves the forward agent and the midfielders. Attackers usually target to control the ball and score a goal, while defenders prevent the opponent from scoring. Team formation is shaped by the positions of defender or midfielder agents. These agents calculate their next positions based on a distributed Voronoi cell construction which is the main contribution of this work. Voronoi cell decomposition method is previously applied to robot soccer. However, our method differs from earlier work in the construction of cells both as the calculation and the way the overall diagram is formed. First, there is no supervision of cell construction which is performed in a completely distributed manner. Second, our method neither relies on communication nor need a high communication bandwidth among agents. However, if communication is available, this channel is also used to improve the solution quality. Since the approach is not heavily dependent on explicit communication, failures in communication could also be handled. This feature is especially useful in real-world settings. Another contribution of our method lies in the automatic online determination of targets for agents. Therefore, there is no need to previously determine special formations.

This paper's structure is as follows: Section 2 reviews earlier work in the field. Section 3 presents the main team strategy for robotic soccer games and the proposed procedures: the case-based group formation approach and the distributed adaptive formation. Experimental results and performance analysis of the approach in terms of ball possession ratio and use of communication are presented in Section 4. Finally, Section 5 concludes the paper.

\section{RELATED WORK}

Dynamic team formation problem has been investigated in earlier work for both humanitarian and military applications (Balch and Arkin, 2000; Stone and Veloso, 1999). Successful results of these works have been used in RoboCup environments as well (Candea, Hu, Iocchi, Nardi and Piaggio, 2011; Nair, Tambe and Marsella, 2003; Röfer, 2003). Multirobot coordination approaches used in RoboCup environments mostly rely on continuous communication among agents. However, RoboCup 3D SSL doesn't provide a supervisor and communication among agents is limited.

Several team formation algorithms were applied in RoboCup soccer competitions (Dashti et al., 2006; Nakanishi, Murakami and Naruse, 2008; Reis, Lau and Oliviera, 2001; Ros, Arcos, de Mantaras and Veloso, 2009). Dashti et al. (2006) use Voronoi cells to position and distribute players in the field for RoboCup 2D SSL. With this method, each agent calculates its own Voronoi cell and moves to the center of its own cell. Dynamically calculating the 
cells ensures the agents to scatter throughout the field. After the distribution is achieved, agents move to better positions by attraction vectors and calculate their Voronoi cells dynamically to be distributed in the field again. Even though this method is efficient for fast-moving 2D soccer agents, slower humanoid agents in 3D SSL should maintain proximity to each other in order to gain control of the ball quickly when it is lost. Therefore, distributing the agents throughout the field may not result in the desired outcome in 3D SSL.

Nakanishi et al. (2008) propose Dominant Region (DR) diagrams to create a formation. DR diagrams look like Voronoi diagrams, but the required calculation is based on the arrival time of all agents to their future positions. Each agent forms its region based on an area where it can reach to faster than its teammates. Players can move in their regions in order to be positioned on the field. With this approach, the agent which is closest to the ball approaches to the ball and the others can follow it while staying in their dominant regions. This method can be useful in 3D humanoid soccer but needs a supervisor or a high communication bandwidth among the agents to calculate a general DR diagram. Therefore, it may not be suitable for environments with limited communication.

Situation Based Strategic Positioning (SBSP) (Reis et al., 2001) is another team formation approach which uses game information including the current position of the agent and its current role, the selected formation for the team and the positions of others. Maintaining this information, agents move to their positions according to their roles. This method requires dynamically assigning roles to the agents during the game. SBSP suffers from a complicated rule-based algorithm to reach a final formation in non-deterministic and noisy environments like RoboCup 3D SSL due to the computation requirements.

Forming groups in the team usually results in better team performance. Ayanian, Kumar and Koditschek (2011) introduce a method which coordinates the agents within each group by explicit communication. Forming groups with optimal number of agents can prevent unnecessary crowds. Therefore, agents in different groups can achieve multiple tasks which might help completing those tasks faster. While inter-group communication is kept limited, intra group communication demands are high.

Ros et al. (2009) propose a Case-Based Reasoning (CBR) method to position the agents. Cases represent both the action sequences and the formations by keeping the game situations including positions of the agents, game time, current score etc. CBR is an applicable formation method but in some of the CBR methods, the cases should be handcoded before and usually the number of them is limited. Some of the CBR approaches update their case libraries in runtime to modify cases but this is a costly process.

Our approach uses Voronoi cell decomposition as in Dashti et al. (2006) but differs from this approach by its initial frame construction and its adaptability based on the ball location. The objective is not spreading out all players on the field but constructing a formation around the ball to easily possess it whenever possible.

\section{DISTRIBUTED TEAM STRATEGY}

The proposed distributed team formation strategy involves four sequential processes to determine a target for an agent. Figure 1 presents the main modules for the team strategy. Initially two groups, namely attackers and defenders, are formed by using a Case-Based group formation strategy (Aamodt and Plaza, 1994). The role of each agent is determined based on these groups. The attackers group involves the forward and the midfielder agents while the defenders group involves only the defender agents. Our adaptive formation method relies on the construction of Voronoi cells, which are generated distinctly by each agent that has the role of midfielder or defender. The centers of these cells form the initial targets for these agents. Target locations are finalized by applying Potential Fields Method (Arkin, 1998) for obstacle avoidance and path planning. Because controlling the ball is crucial in soccer, its location is used in cell initialization and forming groups. All agents except goalkeeper continuously send their time costs to control the ball and they decide on the forward agent role according to the incoming cost information and a selfcalculation.

The Partial Fourier Series (PFS) model is used as the motion model for our RoboCup 3D SSL beeStanbul team software (Asta and Sariel-Talay, 2011). Different types of body motions, including straight walks (forward, back, diagonal and side walk), inward turn, outward turn, rotate, kick and stand-up are available for agents. Based on the assigned role of an agent, the corresponding planner is activated. Each plan has a set of behaviors which 
activate a set of motions. Figure 2 shows the decomposition of an example plan (dribble-to-goal) for an agent that has the forward role.

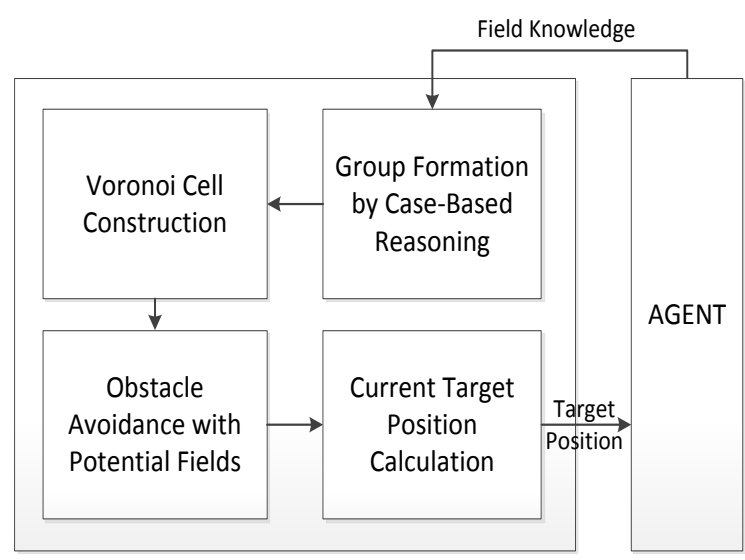

Figure 1: General structure of the distributed team formation method.

As described in Section 1, goalkeeper positions itself around the defense area regardless of the team formation. The forward agent (i.e., the closest agent to the ball) always targets to possess the ball. While goalkeeper is a static role assigned to an agent, the remaining agents switch between the other roles according to their time costs to reach to the ball.

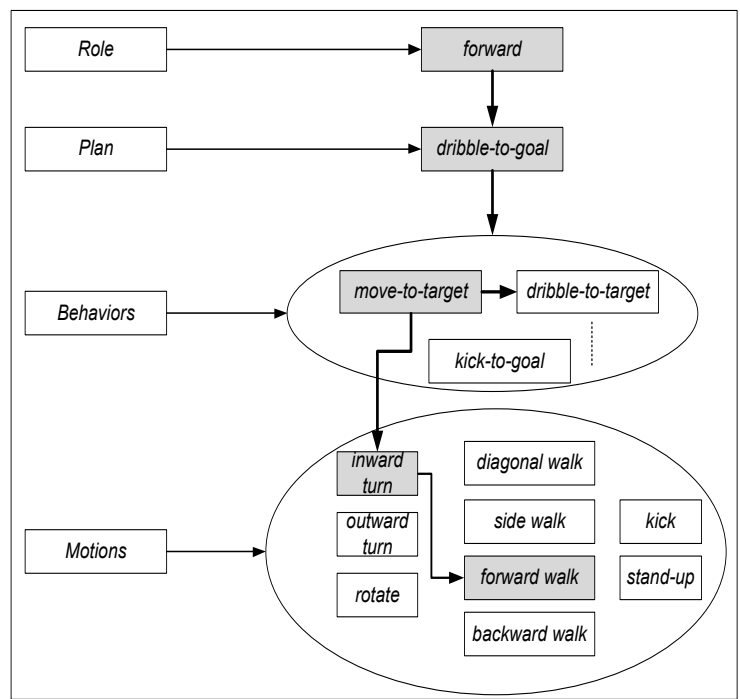

Figure 2: The decomposition of an example plan for an agent that has the forward role. Lower level components are hierarchically activated by selection at a higher level. At the lowest level, primitive actions are selected and the corresponding motion commands are sent to the server.

\subsection{Case-Based Group Formation}

The current setup of the RoboCup 3D SSL involves nine team players in each team. A single player is assigned to the goalkeeper role. Our strategy divides the rest of the team into two groups, namely, defenders and attackers, for offensive and defensive strategies. Attackers are formed for constructing an attacking formation around the ball and scoring a goal whenever possible. This group involves the forward agent and the midfielders which usually target to control the ball and score a goal. Defenders are formed for blocking and constructing a defensive obstacle against the opponent team. This strategy prevents the opponent team from scoring.

We use a case-based group formation method (Aamodt and Plaza, 1994) to determine the number of defender agents and midfielder agents dynamically. Since two agents are assigned to the goalkeeper and the forward roles, the remaining seven agents are to be assigned to these roles. Instead of using a predetermined number for these roles, a case-based method is applied to determine the best separation.

The current game score and the positions of agents and the ball are considered in the problem description of cases. The general structure of cases is shown in (1). Each case corresponds to a certain number of agents for defenders and midfielders. For example, if the team is losing in the middle of the game, more players could be assigned as midfielders to tie the game with more attacker agents while taking the risk of conceding a goal.

$$
\begin{aligned}
& \text { Case }=\{\text { Ball Position, Game Score, } \\
& \text { Agent Positions, Number of midfielders, } \\
& \text { Number of defenders }\}
\end{aligned}
$$

The case library initially involves 12 predetermined cases which are allowed to be modified in runtime according to the success of applying them.

The maximum bandwidth for RoboCup 3D SSL agents is $20 \mathrm{~B}$ for each cycle which can be used by a single agent. This communication channel can be used by a single agent to send role assignments for group formations. We have selected the goalkeeper as the captain of the team because it has the widest line of sight of the field. The time period to communicate is shared effectively by each agent. The goalkeeper is responsible to send group formation messages according to the results of the case-based grouping method while other agents send their costs to reach at the ball position. If agents fail 
to communicate with each other, they behave according to their field knowledge and observations.

Figure 3 shows each agent's role selection strategy and its decision for joining to a group. The agent that is closest to the ball assigns itself the forward role and directly looks for ball possession to score against the opponent. The other agents in the attackers group take midfielder role and follow the forward agent in a close proximity for handling passes or failures. The defenders position themselves at a distance behind the ball to defend the goal. Goalkeeper continuously sends the ball position and the numbers of the teammates that are going to be in attackers group according to the case-based grouping method. If a player hears its number in the latest message string, it positions itself as one of the attackers. Otherwise, it takes the defender role. If the goalkeeper fails to send messages to the others due to falling down or any other reason, it sends a failure message to inform them. If the other players don't hear any messages or hear the failure message, they act on their behalf through observation. In this case, five players closest to the ball assign themselves the attackers group and three players the defenders group in a static manner. If a player observes five teammates that are closer to the ball than itself, it acts like a defender and uses a defensive Voronoi cell calculation method. In the opposite situation, it acts as either the forward agent or a midfielder agent. Midfielder agents calculate Voronoi cells to determine their targets while the forward agent directly targets the ball.

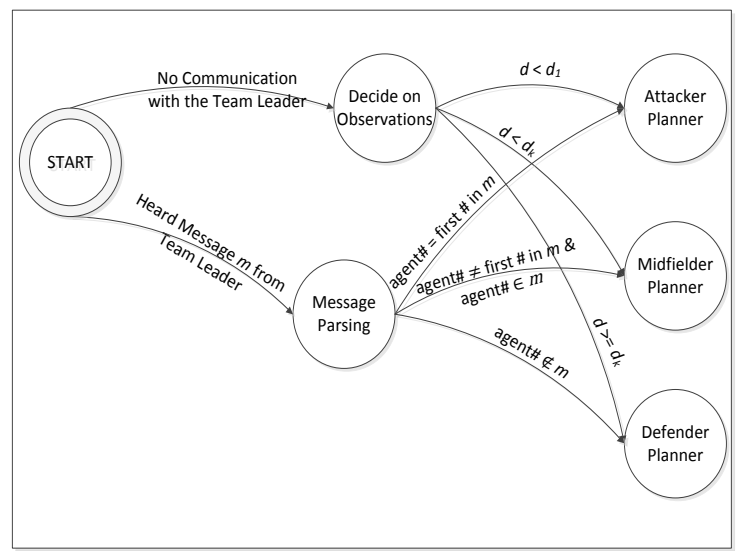

Figure 3: FSM for agents' group formation behavior according to the team captain messages or observations. $\mathrm{d}_{\mathrm{i}}: \mathrm{i}^{\text {th }}$ lowest Euclidean distance between the ball and the agents in the viewpoint, d: Euclidean distance between the agent and the ball, k: the maximum number of attackers.

\subsection{Target Selection by Adaptive Voronoi Cell Construction}

The midfielder and defender agents need to position themselves for maintaining close proximity to the forward agent and defending the goal respectively. This is accomplished by a distributed Voronoi cell construction approach in which each agent calculates its own cell independent from that of the others. Therefore, every agent has a different shaped cell and these can overlap.

In conventional Voronoi diagram computation, Fortune Algorithm (FA) (de Berg, van Kreveld, Overmars, and Schwarzkopf, 2000) is used. Our approach differs from FA in the construction of the final cell. The initial cell is constructed by considering the ball location and then, iteratively narrowed down to get the final cell for the agent. In FA, the lines that construct the cells are perpendicular bisectors of the line segment between teammate locations. In our approach, a line from the corresponding teammate position parallel to the perpendicular bisector is used. The main procedure for our distributed cell construction approach for each agent is given in Algorithm 1.

After constructing the cell for itself, each agent determines the center of the cell as its new target. Agents become closer to each other by using this strategy, which is more beneficial for attacking in soccer. However, RoboCup 3D SSL league have some rules to prevent crowding an area with multiple agents. According to these rules, a player is repositioned out of the field if it is in a circle that has a radius of 1 meter with two other teammate players. In order to overcome the situation where there is a teammate closer than $2 \mathrm{~m}$, the cell is adjusted to keep at least $1 \mathrm{~m}$ distance from that teammate. Applying these alterations on the construction of a cell, the distance to any teammate is guaranteed to be greater than $1 \mathrm{~m}$. In RoboCup 3D SSL, each agent has a 120 degrees angle of view. Therefore, agents only consider the positions of teammates they can see and the ball's last seen position to construct their Voronoi cells. Euclidean distance is used for distance calculations. (2) shows the distance formula for two coordinates $\left(A\left(x_{1}, y_{1}\right), \mathrm{B}\left(x_{2}, y_{2}\right)\right)$ that is used in Algorithm 1.

$$
\operatorname{dist}(A, B)=\sqrt{\left(x_{1}-x_{2}\right)^{2}+\left(y_{1}-y_{2}\right)^{2}}
$$




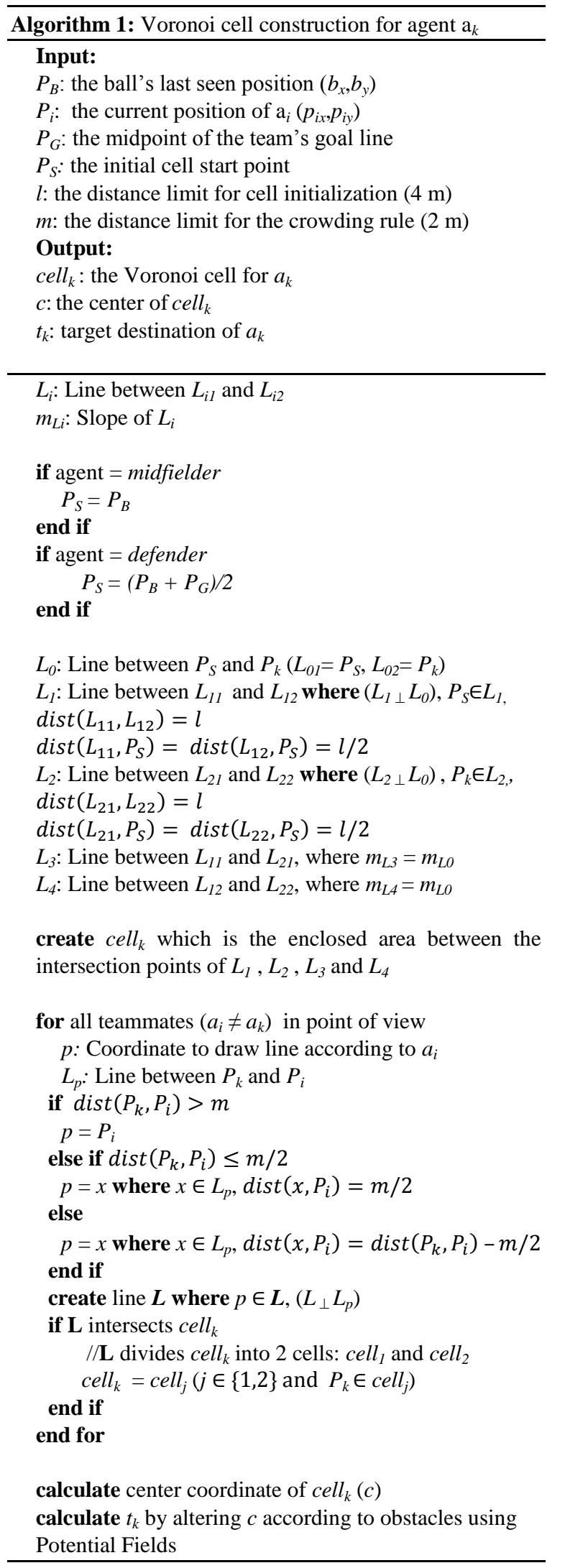

Algorithm 1 is used for both midfielders and defenders. Defenders create their cells with the same algorithm, but their initial cell is calculated according to the midpoint of the line connecting the ball position and the center of the team's goal position while midfielders use the ball location.

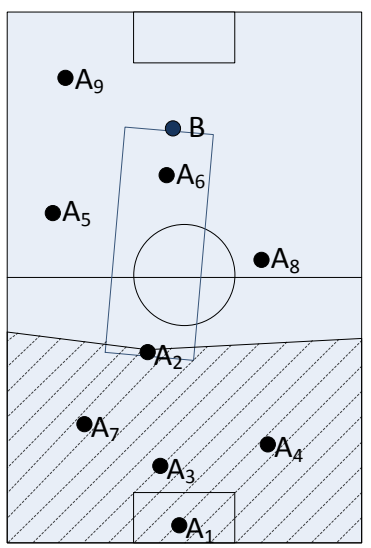

(a)

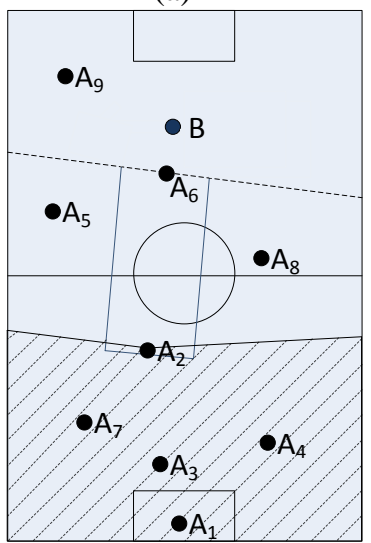

(c)

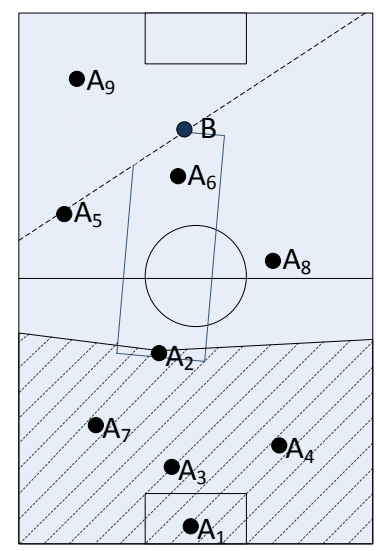

(b)

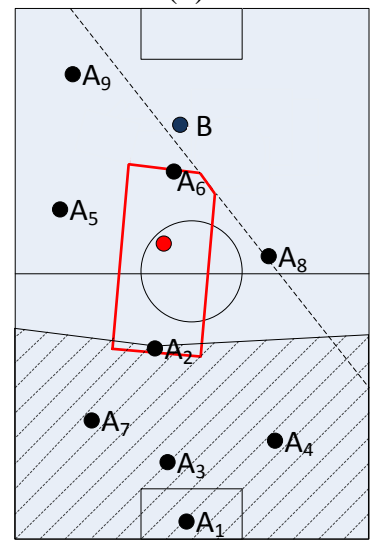

(d)

Figure 4: Step-by-step calculation of the Voronoi cell for $a_{2}$. (a) construction of the initial cell according to the ball position, (b) cell iteration due to the intersections with $\mathrm{a}_{5}$, (c) cell iteration due to the intersections with $a_{6}$, (d) the final cell for $\mathrm{a}_{2}$. The corresponding target position is marked with a red point.

The time complexity of the algorithm is $\mathrm{O}\left(n^{2}\right)$ where $n$ is the number of agents in the team. Figure 4 shows the iterations for calculating the final cell and the corresponding target as the center of this cell for agent \#2 $\left(a_{2}\right)$, which is a midfielder and draws its initial cell according to the ball position. As mentioned before, only teammates in the viewpoint of the agent are considered. The area that is out of $a_{2}$ 's point of view is shown as the shaded area. Figure 4 (a) shows the initial cell construction by considering the ball position $\left(P_{b}\right)$. In Figure $4(\mathrm{~b})$, 
(c), and (d), the cell is modified according to the locations of $a_{5}, a_{6}, a_{8}$ and $a_{9}$, respectively. The line for $a_{9}$ doesn't have any intersection points with the current cell, so it doesn't make any changes in the cell. The final Voronoi cell of $a_{2}$ is shown with the red frame and the center of that cell is marked with a red point in Figure. 4 (d).

Agents continually form their Voronoi cells and move toward their targets. Due to the distributed calculation of cells, a complete diagram is not formed. Cells of different agents may overlap in some situations, but the relevant precautions taken to overcome the crowding rule and the Potential Fields Method ensures that the targets are not too close to each other. This approach also protects agents from collusions.

\section{EXPERIMENTAL RESULTS}

Two sets of experiments are set to analyze the performance of our proposed team strategy. rcssserver $3 d$ is used for the simulation, which is the official server software for RoboCup 3D SSL competitions and RoboViz (Stoecker and Visser, 2011) as a visualization tool.

In soccer game, keeping possession of the ball is one of the key factors for scoring a goal. Our first experiment targets to analyze this issue and the average position of the ball in the field. Ball position fields are determined by dividing the $21 \times 14 \mathrm{~m}$ field horizontally into 3 equal areas (defense, midfield and forward) each $7 \times 14 \mathrm{~m}$. The area next to the team's goal is called the defense area, the area next to the opponent's goal is called the forward area and the area between these two fields is the midfield area. The proposed method is compared to our earlier method Situation Based Strategic Positioning (SBSP) that we used in RoboCup German Open 2011 competitions, our previous Voronoi cell based method which uses a static grouping strategy instead of case-based grouping and DPVC method (Dashti et al., 2006). In DPVC, Voronoi cells are used to scatter the agents throughout the field. In SBSP, each agent has a predetermined role and they shape formations according to predefined positions around the ball based on their roles. All the approaches are applied on the latest motion model of our team.

RoboCup 2011 binary of Nao Team Humboldt (Burkhard et al., 2011) is used as an opponent because the motion model of Nao Team Humboldt is close in speed to that of our PFS model. Also Nao Team Humboldt has a successful defensive team formation which blocks the opponent. We run 10 games for each method against Nao Team Humboldt. A snapshot is shown in Figure 5 from an instance during these games where the blue agents are from beeStanbul team and the red agents from Nao Team Humboldt. This figure also illustrates the Voronoi cell of each midfielder agent in beeStanbul. The agent closest to the ball assigns itself the forward role while the rest of them are assigned to the midfielder role in the attackers group. As can be seen from the figure, the Voronoi cells of midfielders may overlap as it is allowed. However, target positions as the centers of these cells are always different if agents see each other.

Table 1 shows the overall results of all methods. These results illustrate that, the new approach outperforms our previous approaches and DPVC in terms of ball possession, keeping control of the ball and carrying the ball to the opponent's area. Our previous approach that uses Voronoi cells combined with a static grouping method also gives good results but using a case-based method for grouping further improves the overall performance. According to these results, the key factors for the success of the proposed team strategy can be listed as the distributed online construction of Voronoi cells and dynamic positioning to the centers of these cells. Even when the forward agent falls over during an attack, by this approach, midfielders maintain close proximity with the forward agent and regain control of the ball. Communication is also used to dynamically form attacker and defender groups. Another advantage of the new approach is the unpredictability of the team strategy as a competitive strategy. There are not fixed formations that can be learned and predicted by the opponent during a game.

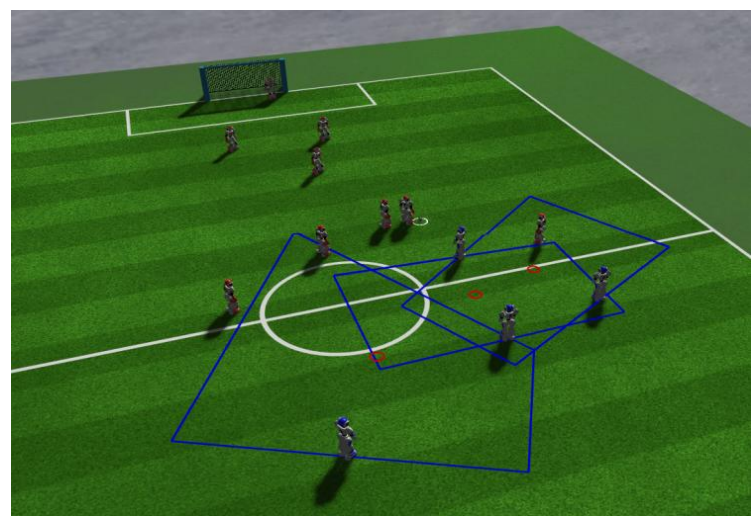

Figure 5: An instance from a game using rcssserver $3 d$ of Simspark for simulation and RoboViz for the visualization. Blue polygons indicate the cells of the agents and red circles indicate their centers. 
Table 1. Comparison among the methods used in beeStanbul team software against Nao Team Humboldt in terms of ball possession and ball position

\begin{tabular}{|c|c|c|c|c|}
\hline & $\begin{array}{l}\text { Distributed Voronoi } \\
\text { Approach with case- } \\
\text { based grouping }\end{array}$ & $\begin{array}{l}\text { Distributed Voronoi } \\
\text { Approach with static } \\
\text { grouping }\end{array}$ & DPVC & SBSP \\
\hline $\begin{array}{l}\text { Ball Possession } \\
\text { Ratio }\end{array}$ & $\begin{array}{c}53.23177 \% \\
(\sigma=0.04441)\end{array}$ & $\begin{array}{c}52.92768 \% \\
(\sigma=0.08248)\end{array}$ & $\begin{array}{c}47.83140 \% \\
(\sigma=0.07796)\end{array}$ & $\begin{array}{c}50.45958 \% \\
(\sigma=0.06877)\end{array}$ \\
\hline $\begin{array}{l}\text { Ball in Own } \\
\text { Area }\end{array}$ & $\begin{array}{c}15.95018 \% \\
(\sigma=0.04423)\end{array}$ & $\begin{array}{c}17.11815 \% \\
(\sigma=0.11013)\end{array}$ & $\begin{array}{c}27.76341 \% \\
(\sigma=0.13851)\end{array}$ & $\begin{array}{c}37.04670 \% \\
(\sigma=0.18277)\end{array}$ \\
\hline Ball in Midfield & $\begin{array}{c}37.48712 \% \\
(\sigma=0.08912)\end{array}$ & $\begin{array}{c}38.37606 \% \\
(\sigma=0.14637)\end{array}$ & $\begin{array}{c}31.74228 \% \\
(\sigma=0.11037)\end{array}$ & $\begin{array}{c}33.22326 \% \\
(\sigma=0.10827)\end{array}$ \\
\hline $\begin{array}{c}\text { Ball in } \\
\text { Opponent Area }\end{array}$ & $\begin{array}{c}46.56270 \% \\
(\sigma=0.11047)\end{array}$ & $\begin{array}{c}44.50579 \% \\
(\sigma=0.18259)\end{array}$ & $\begin{array}{c}40.49431 \% \\
(\sigma=0.13937)\end{array}$ & $\begin{array}{c}29.73004 \% \\
(\sigma=0.24710)\end{array}$ \\
\hline
\end{tabular}

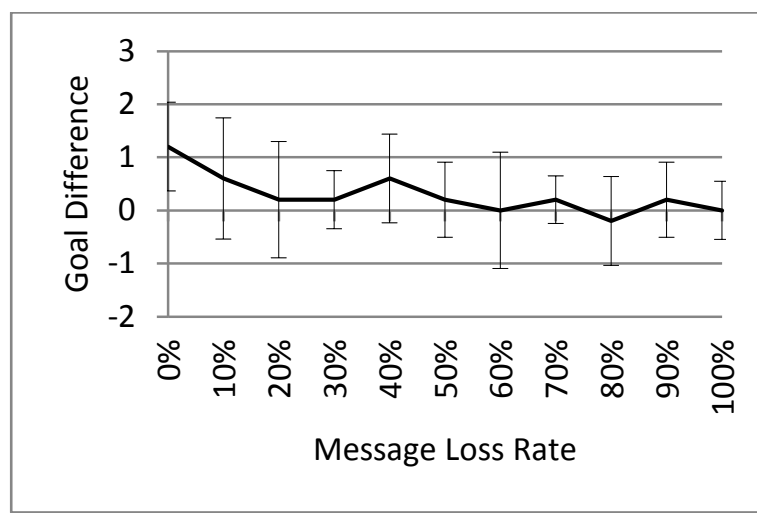

(a)

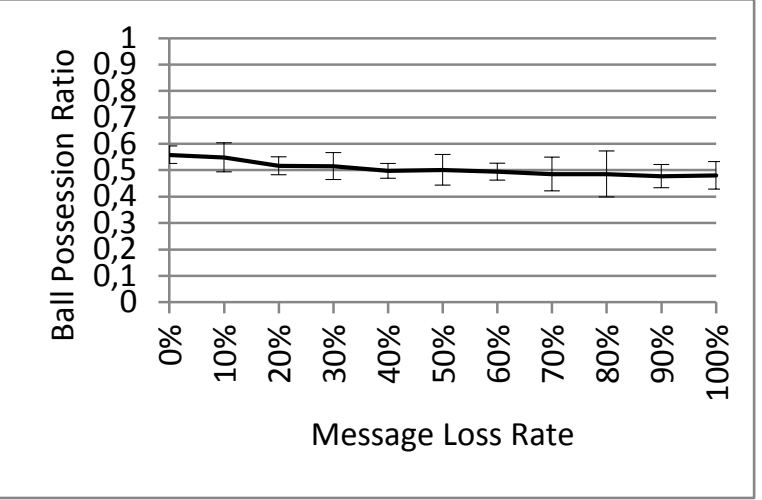

(b)

Figure 6: Test results for each message loss rate presented as the averages of 5 games against Nao Team Humboldt. (a) Average goal difference (positive values show the scores in favor of our team) (b) Average ball possession ratio of our team.

As expected, the performance of DPVC is better than that of SBSP in terms of carrying the ball to the opponent's area due to the dynamism. In SBSP, on the other hand, predetermined formations are easy to be predicted by the opponent in a later time step during the game. However, ball possession performance of DPVC is worse than that of SBSP because it scatters the agents throughout the field. In that case, if the motion model of the agents is not fast enough, they may not responsively regain the control of the ball when it is lost.

In the second set of experiments, we measure the performance of our method for different message loss rates. In rcssserver $3 d$, a team is allowed to send a message periodically in 0.06 seconds. In our current implementation, we use all the available messaging periods in order to perform better. In this experiment, we manually switched off communication based on the message loss rate to simulate communication failure. The reported results indicate that our method is robust to communication failures for most of the instances. Even for no communication cases, agents can still make decisions and calculate their Voronoi cells based on observations and they position themselves to appropriate target locations for maintaining an efficient formation. This is achieved by the distributed implementation of Voronoi cell construction. However, as expected, ball possession performance is degraded gradually with the worst value $47.7 \%$. 


\section{CONCLUSION AND FUTURE WORK}

We have presented an adaptive team formation method for RoboCup 3D SSL. Our proposed Voronoi Diagram based formation generation method requires less computational cost than the standard Voronoi Diagram generation. The ball position is also taken into account during these calculations. We combined our Adaptive Voronoi Diagram with a Case-Based group formation method controlled by an agent (i.e., goalkeeper) through explicit communication. The agents are divided into defender and attacker groups according to this agent's messages. We compared our method against our previous Voronoi cell approach that is combined with a static group formation algorithm, and earlier methods. The results illustrate that the new approach outperforms the other approaches. In our future work, we plan to change the team leader to determine the group behaviors dynamically in run time. In order to provide a better formation, we plan to assign the captain role to the agent that has the best angle of view on the field dynamically.

\section{REFERENCES}

Aamodt A. and Plaza E., 1994. Case-Based Reasoning: Foundational Issues, Methodological Variations, and System Approaches. Artificial Intelligence Communications 7:1, pp. 39-52.

Aldebaran Robotics Official Website, 2011. http://www.aldebaran-robotics.com/

Arkin, R., 1998. Behavior-Based Robotics. MIT Press, Cambridge, Massachusetts.

Asta, S., Sonmez T., Ulusoy O., Alimoglu A., Ersen M., Sozmen O. and Sariel-Talay S., 2011. beeStanbul RoboCup 3D Simulation League Team Description Paper 2011. RoboCup 2011, Istanbul 5-11, July, 2011.

Asta, S. and Sariel-Talay S., 2011. Nature-Inspired Optimization for Biped Robot Locomotion and Gait Planning. The 6th European Event on Nature-inspired Techniques in Scheduling, Planning and Timetabling, Torino, Italy, 27-29 April, 2011, pp. 434-443.

Ayanian, N., Kumar, V. and Koditschek, D., 2011. Synthesis of Controllers to Create, Maintain, and Reconfigure Robot Formations with Communication Constraints. Springer Tracts in Advanced Robotics, Volume 70/2011, pp. 625-642.
Burkhard, H.D., Krause, T., Mellmann, H., Ritter, C.N., $\mathrm{Xu}$, Y., Scheunemann, M., Schneider, M. and Holzhauer, F., 2011. NaoTH 2011 The RoboCup Team of Humboldt-Universitat zu Berlin. RoboCup 2011, Istanbul 5-11, July, 2011.

Candea, C., Hu, H., Iocchi, L., Nardi, D. and Piaggio, M., 2001. Coordination in multi-agent RoboCup teams. Robotics and Autonomous Systems, Volume 36, Issues 2-3, pp. 67-86.

Balch, T. and Arkin, R., 2000. Behavior-Based Formation Control for Multirobot Teams. Proceedings of Fourth International Conference on Multi-Agent Systems, pp. 363-364.

Dashti, H.T., Aghaeepour, N., Asadi, S., Bastani, M., Delafkar, Z., Disfani, F.M., Ghaderi, S.M., Kamali, S., Pashami, S. and Siahpirani, A.F., 2006. Dynamic Positioning based on Voronoi Cells. RoboCup 2005. LNCS (LNAI), vol. 4020, pp. 219-229.

de Berg, M., van Kreveld, M., Overmars, M. and Schwarzkopf, O., 2000. Computational Geometry. Springer-Verlag, New York, 2000.

Nair, R., Tambe, M. and Marsella, S., 2003. Team Formation for Reformation in Multiagent Domains Like RoboCupRescue. RoboCup 2002: Robot Soccer World Cup VI, Lecture Notes in Computer Science, Volume 2752/2003, pp. 150-161.

Nakanishi, R., Murakami, K. and Naruse, T., 2008. Dynamic Positioning Method Based on Dominant Region Diagram to Realize Successful Cooperative Play. RoboCup 2007: Robot Soccer World Cup XI Lecture Notes in Computer Science, Volume 5001/2008, pp. 488-495.

Reis L.P., Lau N., Oliveira E.C., 2001. Situation Based Strategic Positioning for Coordinating a Team of Homogeneous Agents. Springer's Lecture Notes in Artificial Intelligence, Vol.2103, Berlin, pp. 175-197.

Ros, R., Arcos, J.L., de Mantaras, R.L. and Veloso, M., 2009. A case-based approach for coordinated action selection in robot soccer. Artificial Intelligence, v.173 n.9-10, pp. 1014-1039.

Röfer, T., 2003. An Architecture for a National RoboCup Team. RoboCup 2002: Robot Soccer World Cup VI, Lecture Notes in Computer Science, Volume 2752/2003, pp. 417-425.

Simspark Official Website, 2011. http://simspark.sourceforge.net/

Stoecker, J. and Visser, U., 2011. RoboViz: Programmable Visualization for Simulated Soccer. RoboCup 2011, Istanbul 5-11, July, 2011.

Stone, P. and Veloso, M., 1999. Task Decomposition and Dynamic Role Assignment for Real-Time Strategic Teamwork. Intelligent Agents V: Agents Theories, Architectures, and Languages, Lecture Notes in Computer Science, Volume 1555/1999, pp. 293-308. 